Canadian Oncology

Nursing Journal

Revue canadienne

de soins infirmiers

en oncologie

Volume 28, Issue 2 • Spring 2018

elSSN: 2368-8076 


\section{Partenariat entre patients, leaders en soins infirmiers et chercheurs : résultats d'une stratégie web de transfert des connaissances pour planifier le congé d'hôpital et faciliter les transitions au cours des soins oncologiques}

par Hélène Lefebvre, Isabelle Brault, Odette Roy, Marie-Josée Levert, Dan Lecocq, Maryse Larrivière, Michelle Proulx, en collaboration avec André Myre, André Néron, Caroline Plante, Claudine Tremblay, Hélène Lauzon, Irène Leboeuf, Janique Beauchamp, Johanne Déry, Karine LeBreton, Louise Patenaude, Luther Germain, Maryse Carignan, Nadia Maddalena et Robin Gagnon

\section{RÉSUMÉ}

Un projet de recherche a réuni des patients partenaires, des leaders en soins infirmiers de six milieux cliniques du Québec ainsi que des chercheurs en vue de développer et tester un outil web, le Portail d'échange des savoirs (PES), afin d'améliorer les pratiques de planification des congés et les transitions au cours des soins oncologiques. Le projet a débouché sur la création d'un PES axé sur l'oncologie ouvert à toute la francophonie. Il en a aussi émergé une stratégie novatrice de transfert des connaissances (TC) prenant appui sur le PES et alimentée par le travail collaboratif des différents partenaires, parmi lesquels les patients ont tenu un rôle essentiel. Les résultats ont mis en lumière l'importance, pour la recherche en santé, de donner la parole à des patients partenaires dans le cadre d'une étroite collaboration avec des cliniciens et des chercheurs, pour que les pratiques cliniques soient mieux adaptées aux besoins réels des patients et de leurs proches.

Mots clés : soins infirmiers, oncologie, technologies de l'information et des communications (TIC), transitions au cours des soins, planification des congés, recherche participative, transfert des connaissances (TC).

\section{AU SUJET DES AUTEURS}

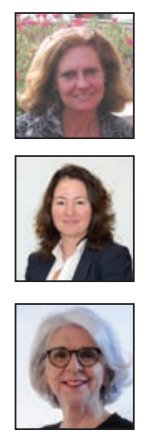

Hélène Lefebvre, inf. aut., Ph.D., Professeure titulaire, Faculté des sciences infirmières, Université de Montréal, C.P. 6128, succ. Centre-Ville Montréal (Québec) H3T 3J7; Courriel : helene.lefebvre@umontreal.ca

Isabelle Brault, inf. aut., Ph.D., Professeure agrégé, Faculté des sciences infirmières, Université de Montréal, C.P. 6128, succ. Centre-Ville Montréal (Québec) H3T 3J7; Courriel :

Isabelle.brault@umontreal.ca

Odette Roy, inf. aut., M.Sc., MAP., Ph.D., Fondatrice et chercheure au Centre d'excellence en soins infirmiers de l'Hôpital MaisonneuveRosemont, fonction qu'elle occupait jusqu'en 2015. Professeure associée à la faculté des Hôpital Maisonneuve-Rosemont, Hôpital Maisonneuve-Rosemont, Professeure adjointe à la faculté des sciences infirmières de l'Université de Montréal C.P. 6128, succ. Centre-Ville Montréal (Québec) H3T 3J7; Courriel : oroy.hmr@ssss.gouv.qc.ca

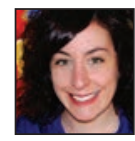

Marie-Josée Levert, Ph.D., Professeure agrégé, Faculté des sciences infirmières, Université de Montréal, Chercheure au Centre de recherche interdisciplinaire en réadaptation du Montréal métropolitain (CRIR), Chercheure pour le Groupe interréseau de recherche sur l'adaptation de la famille et de son environnement (GIRAFE), C.P. 6128, succ. Centre-Ville, Montréal (Québec) H3C 3J7; Courriel : mj.levert@umontreal.ca

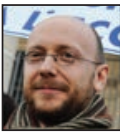

Dan Lecocq, Ph.D. (cand.), Maître de conférences et chercheur, Option "sciences et clinique infirmières ", École de santé publique, Université libre de Bruxelles; Courriel : dan.lecocq@ulb.ac.be

Maryse Larrivière, M.A. (sociologie), Coordonnatrice de projet, Faculté des sciences infirmières, Université de Montréal, C.P. 6128, succ. Centre-Ville, Montréal (Québec) H3C 3J7; Courriel : coordo.riups@gmail.com

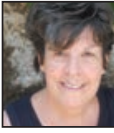

Michelle Proulx, Ph.D. (santé publique), Professionnelle de recherche, Faculté des sciences infirmières, Université de Montréal, C.P. 6128, succ. Centre-Ville, Montréal (Québec) H3C 3J7; Courriel : proulxmichelle@videotron.ca

En collaboration avec :

André Myre, Patient partenaire du projet; Courriel : myre.andre@gmail.com André Néron, patient partenaire; Courriel : andre.neron@ulb.ac.be

Caroline Plante, inf. aut., Conseillère en matière de soins infirmiers, Secteurs hématologie/oncologie, rhumatologie, immunologie et dermatologie Centre hospitalier universitaire Sainte-Justine, Montréal (Québec); Courriel : caroline.plante.hsj@ssss.gouv.qc.ca

Claudine Tremblay, inf. aut., M.Sc.inf., Infirmière-chef des soins palliatifs, responsable CRID et IPO, Programme d'oncologie, Hôpital MaisonneuveRosemont, Montréal (Québec); Courriel : claudinetremblay@ssss.gouv.qc.ca

Hélène Lauzon, M.Bibl., Documentaliste, Hôpital Maisonneuve-Rosemont, Montréal (Québec); Courriel : informationniste.riups@gmail.com

Irène Lebœuf, inf. aut., M.Sc.inf., Conseillère en matière de soins spéciaux en oncologie, Centre hospitalier de l'Université de Montréal, Hôpital Saint-Luc du CHUM, Montréal (Québec); Courriel : irene.leboeuf.chum@ssss.gouv.qc.ca

Janique Beauchamp, inf. aut., Conseillère en soins infirmiers cliniques, Recherche et développement, Direction du développement des pratiques professionnelles, Institut universitaire en santé mentale de Montréal, Montréal (Québec); Courriel : jbeauchamp.hlhl @ssss.gouv.qc.ca

Johanne Déry, inf. aut., Ph.D., Directrice adjointe des soins infirmiers, secteur de la recherche, Centre hospitalier universitaire Sainte-Justine, Montréal (Québec); Courriel : johanne.dery.hsj@ssss.gouv.qc.ca

Karine LeBreton, M.Sc.inf., Infirmière-chef adjointe, Programme-clientèle d'oncologie, Hôpital Maisonneuve-Rosemont, Montréal (Québec); Courriel : Icompagna.hmr @ssss.gouv.qc.ca

Louise Patenaude, Patiente partenaire du projet; Courriel : lanpat@videotron.ca Luther Germain, Patient partenaire du projet; Courriel : Igermain@videotron.ca Maryse Carignan, inf. aut., Conseillère clinique, Direction des soins infirmiers, Associée au programme régional de cancérologie, Centre de santé et de services sociaux de Laval, Laval (Québec); Courriel : mcarignan.csssl@ssss.gouv.qc.ca

Nadia Maddalena, M.Sc.inf., Soins de première ligne, Direction des soins infirmiers, CIUSSS du Nord-de-L'Île-de-Montréal; Courriel : N.Maddalena@ssss.gouv.qc.ca

Robin Gagnon, inf., M.Sc.inf., Directeur du programme de soins infirmiers en oncologie, Centre de santé et de services sociaux de Lanaudière, Centre administratif, Joliette (Québec); Courriel : robin.gagnon29@gmail.com

Auteure-ressource : Hélène Lefebvre, inf. aut., Ph.D. Professeure titulaire, Faculté des sciences infirmières, Université de Montréal, C.P. 6128, succ. Centre-Ville Montréal (Québec) H3T 3J7 Canada; Courriel : helene.lefebvre@umontreal.ca; Tél. : 514 343-2178; Téléc. : 514 343-2306

DOI:10.5737/23688076282102109 


\section{INTRODUCTION}

P our optimiser le continuum de soins et les services oncologiques, l'amélioration de la pratique clinique entourant la planification des congés d'hôpital et les transitions au cours des soins est centrale pour les patients atteints du cancer, les professionnels de la santé, les décideurs et les acteurs politiques (Institut de la statistique du Québec [ISQ], 2014; Loutfi et Laflamme, 2006; ministère de la Santé et des Services sociaux [MSSS], 2013). Malgré les nombreux efforts déployés pour mieux coordonner les soins et services offerts aux patients ayant un cancer, d'importantes lacunes demeurent à plusieurs échelons, notamment dans la planification des congés, les transferts intra- ou inter-établissements, et la transition vers les soins à domicile (ISQ, 2014). Les ressources dont on dispose posent également problème en termes de soutien et de communication entre professionnels, que ce soit aux étapes du triage et de l'investigation, de l'annonce du diagnostic, du traitement ou des soins palliatifs (Ganz, Casillas et Hahn, 2008; Garderet, Olivier, Najman et Gorin, 2006; Hewitt et Simone, 1999; ISQ, 2014; Kantsiper et al., 2009). Ces problèmes sont préoccupants vu le nombre élevé de transitions et de planifications de congé de patients (hospitalisés ou externes) durant leur traitement contre le cancer (Burge, Lawson et Critchley, 2005) et les répercussions possibles sur leur santé et leur bienêtre (Comité consultatif concernant les hospitalisations évitables, 2011; Davidson, Moore, MacMillan et Wiens, 2004; Davies et Batehup, 2011).

Dans la foulée des efforts fournis par la Direction québécoise de lutte contre le cancer (DQC) (Loutfi et Laflamme, 2006), on a cerné différents endroits où on pouvait consolider le continuum des soins oncologiques (avant le diagnostic de cancer et la chirurgie). Le MSSQ (2013) a reconnu l'importance d'améliorer la systématisation des pratiques cliniques entourant la planification des congés et les transitions au cours des soins, des étapes qui comptent pour les patients. Dans ce contexte, le Réseau infirmier, un partenaire de soins (RIUPS) de l'Université de Montréal (UdeM), a mené un projet de recherche collaborative en partenariat intitulé Développer une innovation clinique en oncologie : pour un meilleur continuum de soins et de services pour les patients atteints de cancer. Cette innovation visait à réunir des leaders en soins infirmiers oncologiques (et leur équipe multidisciplinaire) issus de milieux cliniques du Grand Montréal avec des patients partenaires et des chercheurs avec qui ils avaient accepté de combiner leur expertise en vue de développer et tester un outil web interactif, le Portail d'échange des savoirs (PES) (Lefebvre et al., 2015). La démarche avait pour but d'améliorer les pratiques liées à la planification des congés et aux transitions au cours des soins oncologiques. Le projet, basé sur le modèle scientifique proposé par Fixsen et collègues (2005) pour la recherche sur la mise en œuvre, s'est déroulé en deux temps. La première phase, préparatoire, a consisté à déployer l'infrastructure du PES en œuvre et à l'implanter dans les équipes cliniques multidisciplinaires en vue de transformer les pratiques de planification des congés et de transition au cours des soins oncologiques. La deuxième phase, exploratoire, a visé à évaluer les retombées du PES en étudiant : 1) comment il s'est implanté dans les milieux cliniques participants; 2) à quel point l'information, les connaissances et les bonnes pratiques étaient accessibles, 3) comment il a transformé les pratiques de planification des congés et les transitions au cours des soins oncologiques. Nous présentons ici les principaux résultats obtenus à la phase d'évaluation.

\section{CADRE DE TRAVAIL}

Nous avons choisi le modèle scientifique de mise en œuvre multi-niveaux pour orienter notre travail, car il couvre plusieurs phases de mise en œuvre, de l'étape préliminaire d'exploration jusqu'à l'adoption (Fixsen et al., 2005), en plus de proposer divers facteurs qui favorisent le succès d'une mise en œuvre dans une perspective longitudinale. Le modèle se divise en cinq (5) étapes : 1) Exploration et choix (p. ex. évaluer à quel point le projet de mise en œuvre répondra aux besoins du milieu ciblé par le changement); 2) Mise en place du programme (p. ex. s'assurer d'un soutien sur les plans stratégiques, financiers et humains); 3) Projet pilote (p. ex. essai de l'innovation à petite échelle); 4) Déploiement (p. ex. tous les acteurs concernés appliquent l'innovation à grande échelle); 5) Pérennisation (p. ex. intégrer l'innovation dans les politiques et protocoles cliniques des établissements). Aux fins de ce projet, nous n'avons utilisé que les quatre premières étapes du modèle pour encadrer les deux parties de notre projet (mise en œuvre et évaluation).

\section{MÉTHODE}

Ce projet exploratoire a adopté un devis collaboratif (Paillé, 1994) faisant intervenir des partenaires ayant des visions et visées distinctes pour les inviter à participer à une démarche de réflexion et de co-construction continue qui nourrira le développement et l'implantation du PES dans les milieux cliniques. La présence de partenaires devait aider à transformer les pratiques de planification des congés et les transitions au cours des soins oncologiques (Sylvain, 2008).

Structure de gouvernance. Le projet reposait sur une structure de gouvernance à plusieurs paliers, réunissant du personnel administratif de l'UdeM et des représentants officiels, des professionnels et des décideurs des milieux cliniques des établissements partenaires, des patients partenaires du projet, et des chercheurs (figure 1). Léquipe de recherche se composait de quatre chercheurs, quatre professionnels (avec les rôles de coordonnateur, de documentaliste, de modérateur du PES et de professionnel de recherche) et une personne à la maîtrise en sciences infirmières.

Six milieux cliniques ont participé au projet, ce qui nous a permis de couvrir le continuum de soins (primaires, secondaires et tertiaires) et nous a procuré une population diversifiée de patients ( 5 milieux pour adultes et un pour enfants) traités pour divers types de cancers (du sein, du pancréas, leucémie myéloïde aiguë, etc.). Nous avons formé des équipes cliniques multidisciplinaires dans chaque milieu (composées de 4 à 12 cliniciens de diverses disciplines et de gestionnaires) 


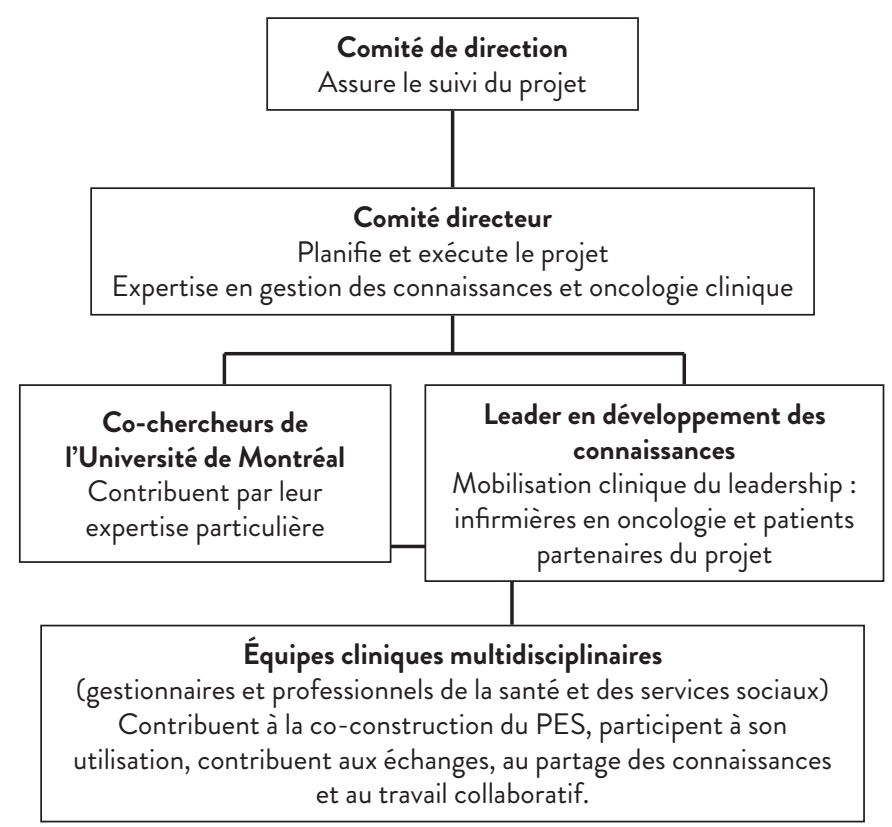

Figure 1. Structure de gouvernance

et leur avons demandé d'utiliser le PES, d'échanger des connaissances et de collaborer ensemble, dans le dessein d'améliorer les pratiques cliniques. Une infirmière leader soutenait chaque équipe. Celle-ci collaborait de près avec différents comités du projet, notamment le groupe d'utilisateurs des connaissances (GUC). Ce groupe comprenait l'équipe de recherche, les leaders en soins infirmiers des milieux cliniques et les patients partenaires du projet. Pendant le développement du PES, le GUC s'est réuni régulièrement pour échanger, résoudre des problèmes rencontrés dans les milieux cliniques, planifier des activités et élaborer des stratégies d'engagement. Huit patients en tout ont été sollicités pour collaborer au projet, et cinq d'entre eux ont été actifs : deux hommes recommandés par la Direction collaboration et partenariat patient (DCPP) de la Faculté de médecine de l'UdeM, un homme recommandé par une infirmière de l'un des milieux cliniques, qui a lui-même recommandé une femme qui avait déjà participé avec lui à un comité d'usagers, et une femme recommandée par une infirmière d'un autre milieu clinique. Les trois autres patients n'ont que peu ou pas participé au projet : l'un a quitté le projet pour retourner aux études, un autre n'a jamais participé, car son équipe ne s'est pas réunie après son recrutement (voir les « Difficultés liées à la mise en œuvre »), et le dernier a participé très peu et à distance seulement (p. ex. en envoyant des textes à ajouter au PES) parce qu'il avait fait un retour au travail.

Collecte et analyse des données. Les données recueillies proviennent de documents papier et électroniques, de l'observation de réunions du GUC, et de 21 entrevues semi-structurées (avec 6 leaders en soins infirmiers actifs tout au long du projet, 1 intérimaire arrivé en cours de projet, 8 gestionnaires d'établissements partenaires et représentants officiels, et 6 patients). D'une durée d'environ 60 minutes, ces entrevues ont été enregistrées, puis transcrites textuellement. Deux membres de l'équipe aguerries en recherche ont classé les données (catégories prédéterminées et nouvelles), qui ont ensuite été validées par les chercheurs du projet. Les synthèses de données par catégories ont ensuite été transmises aux partenaires du GUC, qui les ont annotées, puis retournées aux professionnels qui ont rédigé une première version du rapport de recherche à partir de celles-ci. Des versions successives du rapport ont été discutées aux réunions du GUC jusqu'à arriver à une version définitive satisfaisante. La catégorisation et les analyses ont pris appui sur le modèle scientifique de mise en œuvre de Fixsen et al. (2005) afin d'inscrire le processus de mise en œuvre dans une perspective longitudinale, ainsi que sur le Cadre consolidé pour la recherche sur la mise en œuvre (CFIR) de Damschroder et collègues (2009) en vue de cerner les facteurs favorables ou nuisibles à la mise en œuvre. Les critères habituels de rigueur scientifique dans le paradigme constructiviste ont été respectés (Guba et Lincoln, 1989) : crédibilité (p. ex. triangulation des sources de données, consensus sur l'analyse des données, validations répétées), transférabilité (p. ex. description détaillée du contexte de l'étude), et uniformité (p. ex. indications de tout changement dans la démarche de recherche). Le projet a été présenté aux comités déthique et de recherche des établissements concernés, et les normes de consentement libre et éclairé des sujets, d'accès à l'information et de confidentialité, de prévention des conflits d'intérêts et de protection des sujets de tout préjudice ont été respectées.

\section{RÉSULTATS}

Approche longitudinale de l'étude. Les résultats ont confirmé la faisabilité de l'innovation clinique dans son approche longitudinale de mise en œuvre. Les trois premières étapes du modèle de mise en œuvre de Fixsen et collègues (2005) ont été grosso modo franchies au cours du projet grâce aux efforts fournis à chaque étape. Par exemple, pour la première étape (Exploration et choix), les ententes avec les établissements partenaires concernant la disponibilité des ressources humaines et matérielles (libération du personnel pendant les heures de travail et accès à des ressources matérielles, notamment) ont rapidement été conclues, avant même que le financement du projet soit confirmé, et ont été maintenues à l'obtention des fonds et au durant les travaux. La deuxième étape (Mise en place du programme) a débuté quand les ressources appuyant la mise en œuvre du PES dans les milieux cliniques ont été mises en place (formation d'équipes multidisciplinaires, sélection de postes de travail avec ordinateurs, etc.) et que les réunions du GUC ont commencé. À cette étape, les partenaires ont développé conjointement l'architecture et le contenu du PES. La troisième étape, Projet pilote, a débuté avec le test du PES dans les milieux cliniques, suivie de séances d'inscription (création d'un compte) et de formation sur son utilisation, accompagnées de soutien technique par l'équipe du projet. Cette étape correspond également au lancement des activités entourant le PES dans les milieux cliniques (webinaires, clubs de lecture, forums de discussion, etc.). La quatrième étape, Déploiement, s'est ébauchée à partir du printemps 2015 dans le cadre des travaux collaboratifs du GUC, 
avec pour objectif de développer un outil pour soutenir les patients et leurs proches durant les soins oncologiques (voir aussi les «Résultats du projet »). L'objectif en est double : 1) répondre à toutes les questions et préoccupations des patients à toute étape de leur traitement ou de transition entre deux étapes; 2) aider les professionnels de la santé et des services sociaux (infirmières, médecins, pharmaciens, travailleurs sociaux, psychologues...) et les organisations où ils travaillent à offrir un soutien adéquat.

Facteurs favorisant l'implantation. Un certain nombre déléments ont facilité l'implantation du projet : soutien stratégique de représentants officiels (DQC, MSSS. DCPP de la faculté de médecine de l'UdeM); soutien financier; effet d'émulation (influence positive) provoqué par la collaboration entre les milieux cliniques participants; soutien international (SIRIC : Université de Bordeaux, Bergonié Institute, CHU de Bordeaux; BRIO : recherche professionnelle en cancérologie, Bordeaux, France) établi et maintenu tout au long des activités. Les leaders en soins infirmiers, en tant que personnes d'influence dans leur milieu clinique (p. ex. expertise en oncologie et poste occupé), ont fait preuve d'un fort leadership en s'investissant pendant toute la durée des activités (soutien aux activités liées au projet dans leur milieu clinique, accompagnement dans l'inscription au PES ou aide à l'utiliser, contacts personnalisés pour inviter ou motiver les gens à participer, diffusion du projet à l'interne, etc.). Les gestionnaires des milieux cliniques ont apporté leur contribution dans la mesure de leurs capacités (voir les « Difficultés liées à la mise en œuvre ») à l'échelon administratif, en libérant les employés pendant les heures de travail, en mettant des ressources à leur disposition et en permettant l'accès à des postes de travail avec ordinateurs. Certains ont même fait la promotion du projet auprès des cadres supérieurs de l'établissement ou de membres de leur réseau professionnel. Le leadership des chercheurs et le soutien apporté par la personne assurant la modération du PES (p. ex. en stimulant les échanges dans le forum de discussions, en guidant la collaboration entre les partenaires) et par d'autres professionnels de l'équipe de projet (la coordonnatrice était présente dans les milieux cliniques pour contribuer aux webinaires) figurent également parmi les facteurs favorisant l'engagement. Les équipes du projet ont eu recours à divers moyens pour encourager, améliorer et soutenir la participation (voir Tableau 1. Exemples de stratégies d'engagement).

\section{Tableau 1 : Exemples de stratégies d'engagement}

- Réunions régulières sur place du groupe d'utilisateurs des connaissances (GUC).

- Webinaires, club de lecture, forum de discussion, publication de nouvelles hebdomadaires dans un bulletin.

- Communications personnalisées et rappel des activités.

- Création d'une page Facebook et d'un compte Twitter du PES, en vue d'atteindre les jeunes professionnels plus à l'aise avec les nouvelles technologies ou intéressés par celles-ci.

- Invitation à rédiger des textes et à participer à la production de capsules vidéo.
L'engagement actif des patients partenaires du projet a été essentiel à la mobilisation, leur participation ayant été constante à différents niveaux du projet. Cet engagement s'est traduit par la coproduction de capsules vidéo sur divers thèmes (comme vivre avec le cancer et les traitements, ou encore le rôle du patient partenaire du projet), des présentations durant deux journées d'étude du RIUPS; une participation aux webinaires, aux visites en milieu clinique et aux symposiums et conférences, la rédaction d'articles, et la contribution aux discussions dans le forum du PES. Les patients ont été actifs au sein du GUC (2 à 4 patients étaient présents par réunion), où ils ont contribué aux choix méthodologiques du projet et à la résolution des difficultés liées à la mise en œuvre. Ils ont également participé à la réflexion sur les stratégies de mobilisation des milieux cliniques, à la conception conjointe de l'architecture et du contenu du PES et à la planification des activités greffées à la plateforme. Même si plusieurs gestionnaires et leaders en soins infirmiers se sont dits parfois désarçonnés par les remarques des patients, ils ont apprécié leur franchise. Ils ont souligné que la participation des patients les avait amenés à penser autrement, à prendre conscience des attentes et préoccupations des patients, et avait apporté un nouvel éclairage aux approches de normalisation des soins.

«De toute façon, je pense qu'il est essentiel de connaître le point de vue des patients. Nous avons parfois limpression - tenace, même dans le concept de soins infirmiers! - d'en savoir plus qu'eux. Nous croyons, comme infirmières, connaître les besoins des patients, mais réalisons à un certain moment - du moins, c'est ce que j'ai observé en interagissant avec les patients - que ce dont ils ont parfois réellement besoin est complètement à côté de ce que nous avions cru essentiel pour eux (18). »

«Les patients partenaires du projet ont vraiment été un "plus" pour moi. Nous avions vraiment accès aux deux côtés de la médaille. Nous n'avions plus que des professionnels

\section{Tableau 2 : Exemples d'initiatives des milieux cliniques}

- Mise en ligne de documents d'intérêt par les leaders en soins infirmiers (p. ex. sur l'aide médicale à mourir), documents ensuite échangés avec les collègues.

- Commentaire par certains collègues d'articles scientifiques et ajout de documents provenant d'ailleurs pour nourrir le développement des outils internes.

- Invitations personnalisées à utiliser le contenu du PES pour rédiger des articles ou des fiches sur certains aspects de la pratique qui viendront bonifier le PES.

- Promotion du PES auprès d'infirmières-pivot de divers services, qui ont ensuite choisi de consulter le PES pour préparer des communications.

- Revisionnement d'un webinaire sur le transfert des connaissances (TC) dans un milieu clinique pour les consultants cliniques de divers services.

- Club de lecture établi pour discuter d'articles publiés décrivant un protocole de projet suivi dans le cadre d'une séance d'exploration collective du PES, activité qui a suscité quelques nouvelles inscriptions. 
discutant entre eux de ce qui leur semblait le mieux, nous avions une réaction immédiate. Cela nous a parfois permis de vraiment mieux réaligner et arrimer les choses (22). »

Difficultés liées à la mise en œuvre. Malgré ces conditions gagnantes, deux grandes contraintes nous ont empêchés d'implanter le PES dans les milieux cliniques comme souhaité à l'origine :

1. Près d'un an après le début des activités, cinq des six équipes cliniques multidisciplinaires sont entrées dans une phase de restructuration due à une réforme profonde du réseau de la santé québécois. (Le milieu pédiatrique n'a pas été touché en soi par la réforme, mais les activités de l'équipe ont néanmoins été considérablement ralenties par les problèmes rencontrés dans les autres milieux cliniques participants.) Cette réforme (projet de loi $\mathrm{n}^{\circ} 10$ : Loi modifiant l'organisation et la gouvernance du réseau de la santé et des services sociaux notamment par l'abolition des agences régionales) a mené au remaniement du personnel clinique et administratif, et à l'imposition d'une importante contrainte budgétaire sur tous les établissements de santé du Québec. Touchées par la réforme, les équipes désignées au départ ont donc dû ralentir leurs activités. Le nouveau contexte a graduellement entraîné des changements dans la composition de ces équipes et, dans certains cas, une reconfiguration complète. Il a fallu procéder à plusieurs vagues de recrutement pour reconstituer les équipes, ce qui a entraîné des interruptions.

« Je comprends que le moment n'était pas opportun pour parler aux équipes. Les structures de direction étant chamboulées, presque tous les administrateurs du réseau ont changé de poste. Naturellement, ça complique les choses quand il $y$ a de l'instabilité à l'échelon supérieur, car les administrateurs servaient de relais pour travailler avec les autres équipes (18). »

2. Le réseau informatique désuet des établissements a également représenté tout un obstacle à l'engagement, tout comme la présence de pare-feu, qui sont restés activés tout au long du projet.

«... c'était difficile d'accéder au forum à cause du parefeu. Je pense que, pour les TI, la structure de chaque centre était un beau noud en soi, alors quand on avait besoin du forum, toutes les étapes de sécurité à franchir nous compliquaient passablement la tâche (8). »

En outre, les répondants ont également mentionné comme obstacles potentiels à la participation des professionnels des milieux cliniques la difficulté de faire concorder les visées du projet avec les nouvelles réalités sur le terrain. Parmi ces obstacles figuraient la surcharge de travail, le peu de marge de manœuvre laissée aux administrateurs (dans le contexte de la réforme) pour libérer le personnel pendant les heures de travail, et les retards imprévus de livraison du PES dus à la complexité de sa structure (le choix de l'hébergement et des fonctionnalités du PES dans les limites du budget imparti a été très exigeant). Ces obstacles, selon certains, ont miné l'enthousiasme du début.
Deux modifications importantes au plan initial ont également fait l'objet de discussions : l'ouverture du PES au public (en raison de l'intérêt généré chez les acteurs externes) et la participation accrue de patients partenaires. D'après les répondants, certains membres toujours actifs des équipes désignées se seraient alors sentis exclus et dépossédés de leur rôle d'interlocuteurs principaux. La diversification des sites des tumeurs traitées d'un établissement à l'autre semble aussi, pour certains, avoir compliqué l'établissement de buts et intérêts communs. On a également mentionné comme obstacle potentiel à l'utilisation du PES les difficultés d'utilisation des technologies web en général, et de la plateforme en particulier (navigation difficile pour certains).

Résultats du projet. Malgré ces contraintes, de nombreux résultats positifs ont été relevés.

Un PES à la disposition du secteur de loncologie. Une version française fonctionnelle et opérationnelle du PES était accessible aux professionnels, aux administrateurs, aux patients et à leurs proches dans le domaine de l'oncologie. La plateforme présente plusieurs niveaux de comptes d'utilisateur, des outils sécuritaires de gestion des fichiers et diverses fonctionnalités (module permettant à des partenaires de travailler en collaboration, module de dissémination et vulgarisation des connaissances, module d'ajout de documentations avec renouvellement d'URL). Une section s'adresse tout particulièrement aux patients et à leurs proches, leur donnant la possibilité d'échanger des ressources et des liens qu'ils ont trouvés utiles. On a ajouté une page Facebook et un compte Twitter à la plateforme en vue d'atteindre les jeunes professionnels plus à l'aise avec les nouvelles technologies ou intéressés par celles-ci. La plateforme a été très fréquentée (consultations, utilisation). Les données recueillies du 17 décembre 2014 au 30 août 2015 indiquaient que 155 utilisateurs s'étaient inscrits au départ. Ce nombre est passé à 214 du $1^{\text {er }}$ février 2016 au 31 mai 2016. En février 2017, le PES comptait 234 utilisateurs.

Émergence d'une stratégie innovante de transfert des connaissances. Le projet a fait émerger une stratégie innovante favorisant l'échange de connaissances et la co-construction, ainsi qu'un modèle original de travail collaboratif. La stratégie fait intervenir activement des patients, des leaders en soins infirmiers et des chercheurs dans l'acte de diffusion et de transfert des connaissances grâce au PES et aux multiples activités connexes ( 7 webinaires et clubs de lecture organisés, près de 40 questions posées dans le cadre des forums de discussion, pour un total de 243 interactions, 676 nouvelles publiées dans un bulletin hebdomadaire). La stratégie a pris racine dans les milieux cliniques participants, par le biais de nombreuses activités organisées de l'initiative des soins infirmiers et soutenues par la plateforme (voir Tableau 2. Exemples d'initiatives des milieux cliniques).

Les avantages d'un partenariat entre patients, leaders en soins infirmiers et chercheurs. La stratégie de transfert des connaissances, qui tire ses racines de la pratique quotidienne des infirmières leaders et est alimentée par les commentaires de patients et de chercheurs, a donné un nouveau sens 
au projet. Grâce à diverses activités communes (p. ex. ajout de documents internes par les milieux cliniques; élaboration d'une cadre de travail intégratif pour la trajectoire des soins oncologiques), les partenaires connaissent mieux les difficultés liées à la planification des congés d'hôpital et aux transitions au cours des soins oncologiques. Ils ont ainsi pu savoir et prendre conscience des multiples défis associés à la planification des congés et aux transitions et de l'importance de trouver des solutions. Le partenariat du projet a contribué à briser l'isolement entre les établissements, et a mené à l'élaboration d'un discours commun et à la reconnaissance des diverses philosophies et manières d'administrer les soins.

«Pour moi, ce fut une expérience très positive, parce que j'ai vu ce qu'on faisait ailleurs. Ça m'a permis d'acquérir de nouvelles connaissances, et ça m'a enseigné à mouvrir davantage aux différentes façons de voir les choses et d'interagir avec les patients (22). »

«La co-construction du savoir est une bonne chose. Il semble également qu’une relation étroite se soit développée entre les différents acteurs, comme c'est le cas dans toute communauté de pratique une fois que les gens commencent à se faire confiance et deviennent à l'aise pour échanger. Lorsqu'on dépasse la mentalité du silo et la culture de la compétition, la collaboration se manifeste (9).»

Grâce à la participation active des patients partenaires du projet, les difficultés liées à la planification des congés et aux transitions au cours des soins ont pu progressivement être examinées sous un autre angle. Partant d'une orientation initiale fondée sur les points de vue professionnels et organisationnels, le projet a évolué vers une représentation différente des transitions et de la planification des congés, cette fois vue par la lorgnette des patients atteints du cancer et de leurs proches. Une ébauche d'outil d'accompagnement, actuellement en développement, a pris forme et sera mise à la disposition des patients et de leurs proches.

Les infirmières et les administrateurs interrogés ont mentionné être maintenant convaincus de l'importance d'encourager le partenariat avec des patients dans de futurs projets du RIUPS et dans leurs propres projets de développement conjoint des pratiques cliniques. Les patients partenaires du projet ont eux aussi profité de leur participation (p. ex. en se sentant valorisés pour leur contribution, en pouvant se distancer de leur expérience du cancer, en comprenant mieux le fonctionnement du réseau de la santé).

"Ça m'a beaucoup aidé de comprendre comment fonctionne le réseau de la santé, et plus j'interviens, plus je suis capable de maintenir une distance émotionnelle quand survient quelque chose que je ne veux pas vivre (patient partenaire; 10). »

«C'est sûr que j'ai découvert des choses. C'est un univers que je ne connaissais pas. Au début, je ne savais rien... Je n'ai pas fait carrière dans la santé, je ne connaissais pas ce monde. Ça m'a beaucoup intéressé. Je pensais que ce serait un excellent défi intellectuel pour moi de participer. C'est certainement très gratifiant de penser que nous pouvons contribuer à quelque chose. J'ai trouvé ça très stimulant... Ça avait beaucoup de sens pour moi, et quel que soit le résultat de cette recherche, je souhaite continuer, aller de l'avant et participer à d'autres types de recherche (patient partenaire; 12).»

La promotion du PES. La diffusion du PES, soutenue et alimentée par ses partenaires et le partenariat avec les patients au cœur du projet, a été très bien accueillie dans la francophonie internationale. Une subvention a été accordée au BRIO (recherche professionnelle en cancérologie, Bordeaux) du SIRIC (Université de Bordeaux, Bergonié Institute et CHU de Bordeaux) en France pour un projet de recherche en cancer du sein. Par ailleurs, un RIUPS belge s'apprête à voir le jour.

\section{DISCUSSION}

Ce projet de recherche collaborative intéressera les infirmières des établissements de santé qui souhaitent améliorer le continuum de soins en oncologie. Il a dévoilé le grand potentiel que peut receler une stratégie de transfert des connaissances quand elle s'appuie sur un outil web interactif comme le Portail d'échange des savoirs (PES). Ce portail est soutenu et alimenté par des partenaires actifs, unis autour d'actions communes visant à améliorer les pratiques de planification des congés d'hôpital et de transitions au cours des soins. Une version française du PES est maintenant offerte dans le secteur de l'oncologie à tous les acteurs des établissements de santé, rendant ainsi possible la diffusion des connaissances et des activités d'échange à même de transformer les pratiques cliniques et de contribuer au perfectionnement continu dans un contexte réel. Les cliniciens au chevet des patients, les décideurs du milieu clinique, les cadres supérieurs, les patients, leurs proches, et d'autres sont maintenant en contact direct les uns avec les autres et ont la possibilité d'engager un dialogue en direct ou en différé. Ils ont tous facilement accès aux connaissances et à la documentation interne fournie par les établissements partenaires du projet (outils, guides d'enseignement...) et ont la possibilité de participer virtuellement à un travail collaboratif. À cet égard, le projet est au diapason d'autres projets du secteur de l'oncologie qui font appel à des outils web interactifs. Ces projets portent sur l'échange de données probantes issues de la recherche, et visent à transformer les pratiques en formant des communautés virtuelles de pratique, des réseaux ou des plateformes interactives (Bartonova, 2012; Dunn, Eisen, Wayne et Crighton, 2004; Farrell, la Porta, Gallagher, Vinson et Bernal, 2014; Grau, Grajales, Gene-Badia, Siso et de Semir, 2013; Sanchez et al., 2012).

Même si le projet a profité d'un certain nombre de facteurs favorisant le succès de sa mise en œuvre (Damschroder et al., 2009), ses partenaires ont été confrontés à des défis qui ont entravé leurs efforts de mobilisation des équipes cliniques dans la mesure voulue. En plus des changements dans la composition des équipes désignées en raison du contexte imposé par la réforme du réseau de la santé au Québec, le matériel informatique désuet et la présence de pare-feu dans les milieux cliniques ont été source de frustration et ont limité l'utilisation du PES et de participer aux activités connexes, et ce, pendant toute la durée du projet. Si la communauté scientifique a 
accueilli à bras ouverts les nouvelles technologies de l'information et de communication, les établissements de soins de santé auraient tout à gagner à en faire autant (Ho, Bloch, Gondocz, Elizabeth et Wenghofer, 2004) et à éliminer les obstacles à l'accès (retrait de pare-feu, augmentation de la bande passante...). Un passage à la technologie mobile (comme les tablettes) et un plus grand recours aux outils en ligne (applications web et médias sociaux, notamment) seront essentiels (Centre de pédagogie appliquée aux sciences de la santé, 2013; Marsan, Paré et Wybo, 2012) si on veut que les cliniciens puissent profiter d'un accès facile et rapide aux connaissances.

La difficulté à arrimer les visées du projet aux nouvelles réalités des établissements de soins de santé et des milieux cliniques a également été mentionnée par des infirmières et des gestionnaires quand il a été question pendant les entrevues des obstacles observés ou possibles à la mobilisation (la difficulté à libérer le personnel pendant les heures de travail ou à articuler le projet autour des besoins des milieux cliniques ont notamment été donnés en exemple). Ces contraintes sont recensées dans la documentation scientifique comme des obstacles à la mise en œuvre des changements (Damschroder et al., 2009; Fixsen et al., 2005). Pour pallier ces difficultés à l'avenir, la stratégie de transfert des connaissances du projet devra demeurer flexible, compte tenu des contraintes organisationnelles en santé (p. ex. budget limité). Elle devra promouvoir et soutenir les initiatives spontanées (Cothrel et Williams, 1999) dans les milieux cliniques et s'appliquer à répondre aux divers besoins, préférences et préoccupations des membres inscrits au PES en passant par de multiples canaux (Pan et Leidner, 2003). Elle devra également exploiter l'inventivité de ses partenaires en vue de rejoindre la grande diversité des acteurs des différents établissements, quelle que soit leur discipline (Langelier, 2005).

Ce projet intéressera toute personne à la recherche d'un partenariat avec des patients, une tendance nouvelle dans les organismes de soins de santé, les établissements et les universités (Brett et al., 2014; Centre de pédagogie appliquée aux sciences de la santé, 2013; Domecq, Prutsky, Elraiyah et al., 2014; Iedema et Angell, 2017; South et al., 2016; Woolf et al., 2016). La participation active des patients partenaires du projet,

\section{RÉFÉRENCES}

Bartonova, A. (2012). How can scientists bring research to use: The HENVINET experience. Environmental Health, 11(Suppl. 1), S2.

Brett, J., Stanislas, S., Mockford, C., Herron-Marx, S., ... Suleman, R. (2014). A systematic review of the impact of patient and public involvement on service users, researchers and community. Patient, 7, 387-395.

Burge, F.I., Lawson, B., \& Critchley, P (2005). Transitions in care during the end of life: Changes experienced following enrolment in a comprehensive palliative care program. BMC Palliative Care, 4(3), $1-7$.

Centre de pédagogie appliquée aux sciences de la santé de l'Université de Montréal (CPASS) (2013). Guide d'implantation du partenariat de soins et de services, vers une collaboration optimale entre intervenants et avec le patient. Québec : Université de Montréal, Direction de collaboration et partenariat patient/CPASS. élément innovant essentiel du projet, a été concluante. Ces derniers ont constitué une force motrice suscitant motivation et mobilisation chez les leaders en soins infirmiers et les chercheurs. Leur engagement a été de toute première importance dans les discussions sur la planification des congés d'hôpital et les transitions au cours des soins. Ils apportaient un autre éclairage au projet, de même que sur ses méthodologies et son orientation. Ce type de contribution est valorisé dans la documentation scientifique (Lavoie-Tremblay et al., 2016; South et al., 2016; Woolf et al., 2016).

\section{CONCLUSION}

Le RIUPS a mis en œuvre un projet de recherche clinique collaboratif innovant en partenariat avec plusieurs établissements et patients partenaires. À notre connaissance, il s'agit de l'un des premiers projets de cette nature à avoir été réalisé dont les résultats peuvent s'avérer utiles pour les chercheurs, les cliniciens et les gestionnaires d'organisations en soins de santé. L'équipe a fait preuve de créativité dans l'élaboration d'une stratégie de recherche qui a permis à tous les acteurs de créer un espace d'échange de connaissances scientifiques et expérientielles dans lequel tous se reconnaissaient experts pour ce contexte. Les réunions du GUC se poursuivent en vue de perfectionner l'outil compagnon. Deux patients partenaires du projet sont encore très actifs dans cette démarche. Le RIUPS est d'avis qu'il est essentiel de donner la parole aux patients dans la recherche en santé, et qu'il faut reconnaître leur contribution par une collaboration étroite avec les cliniciens et les chercheurs en vue de mieux adapter les pratiques cliniques aux besoins réels des patients et de leurs proches.

\section{REMERCIEMENTS}

Nous remercions les Instituts de recherche en santé du Canada (IRSC) et la Fondation pour la recherche en santé (FRS) de leur soutien financier.

\section{CONFLIT D'INTÉRÊTS}

Je déclare, de même que mes co-auteurs et collaborateurs au présent manuscrit, que nous ne faisons l'objet d'aucun conflit d'intérêts.

Centre de santé et de services sociaux de Laval. Ensemble en réseau, pour vaincre le cancer. Plan d'action en cancérologie 2013-2015. Toronto : ministère de la Santé et des Soins de longue durée.

Comité consultatif concernant les hospitalisations évitables (2011). Améliorer le continuum de soins. Toronto : ministère de la Santé et des Soins de longue durée. Cothrel, J., \& Williams, R.L. (1999). On-line communities: helping them form and grow. Journal of Clinical Oncology, 3(1), 54-60.

Damschroder, L.J., Aron, D.C., Keith, R.E, Kirsh, S.R., ... Lowery, J.C. (2009). Fostering implementation of health services research findings into practice: A consolidated framework for advancing implementation science. Implementation Science, 450.

Davidson, B.J., Moore, K.N., MacMillan, H., \& Wiens, K. (2004). Patient evaluation of a discharge program following a radical prostatectomy, Urologic Nursing, 24(6), 483-489. 
Davies, N.J., \& Batehup, L. (2011). Towards a personalized approach to aftercare: A review of cancer follow-up in the UK. Journal of Cancer Education, 5, 142-151.

Domecq, J.P., Prutsky, G., Elraiyah, T., ... Murad, M.H. (2014). Patient engagement in research: A systematic review. BMC Health Serv Res, 14(1), 89.

Dunn, J., Eisen, K., Wayne, J., \& Crighton, F. (2004). The creation of a state and national cancer resource web site for patients and professionals. Oncology Nursing Forum, 31(4), 679-682.

Farrell, M.M., La Porta, M., Gallagher, A., Vinson, C., \& Bernal, S.B. (2014). Research to reality: Moving evidence into practice through an online community of practice. Preventive Chronic Disease, 11, 130272 .

Fixsen, D.L., Naoom, S.F., Blase, K.A., Friedman, R.M., \& Wallace, F. (2005). Implementation research: A synthesis of the literature. Tampa Florida: University of South Florida.

Ganz, A., Casillas, J., \& Hahn, E.E. (2008). Ensuring quality care for cancer survivors: Implementing the survivor care plan. Practice Nurse, 24(3), 208-217.

Garderet, L., Olivier, M-P., Najman, A., \& Gorin, N.-C. (2006). L'annonce d'une mauvaise nouvelle. Revue de littérature. Oncologie, 8, HS126-HS131.

Grau, I., Grajales, I.F.J., Gene-Badia, J., Siso, A., \& de Semir, M. (2013). Forum clinic: The shaping of virtual communities to assist patients with chronic diseases. Studies in Health Technology Q Informatics. 183, 271-275.

Guba, E.G., \& Lincoln, Y.S. (1989). Fourth Generation Evaluation. California: Thousand Oaks: SAGE Publications.

Hewitt, M.E., \& Simone, J.V. (1999). Ensuring quality cancer care. Washington, DC, Academy Press, 256 p.

Ho, K., Bloch, R., Gondocz, T., Elizabeth, F., \& Wenghofer, E.F. (2004). Technology-enabled knowledge translation: Frameworks to promote research and practice. Journal of Continuing Education in the Health Professions, 24(2), 90-99.

Iedema, R.A., \& Angell, B. (2015). What are patients' care experience priorities? BMJ Qual Saf, 24(5), 356-359.

Institut de la statistique du Québec (2014). Études et documents. La lutte contre le cancer au Québec et en Ontario : une approche centrée sur le patient. Québec: Gouvernement du Québec. ERP 978-2-550-70445-4

Kantsiper, M., McDonald, E.L., Geller, G., Shockney, L., Snyder, C., \& Wolff, A. (2009). Transitioning to breast cancer survivorship: Perspectives of patient, cancer specialists and primary care providers. Journal of General Internal Medicine, 24, 2, 459-466.
Langelier, L. (2005). Travailler, apprendre et collaborer en réseau. Guide de mise en place et d'animation de communautés de pratique intentionnelles. Québec : Centre de recherche et d'innovation dans les organisations (CEFRIO). ISBN pour le dépôt légal : 2-923278-14-3.

Lavoie-Tremblay, M., O'Connor, P., MacGibbon, B., \& Fréchette, J. (2016). The experience of patients engaged in co-designing care processes. The Health Care Manager, 35(4), 284-293.

Lefebvre, H., Brault, I., Levert, M-.J., Roy, O., ... Larivière, M. (2015). Une innovation pour un meilleur continuum de soin en oncologie : développement et implantation d'un portail web d'échange des savoirs. Revue francophone internationale de recherche infirmière, 1-10. doi:10.1016/j.pec.2008.07.016

Loutfi, A., \& Laflamme, B. (2006). Rapport d'activités 2005-2006. Direction de lutte contre le cancer. Toronto : ministère de la Santé et des Soins de longue durée. ISBN-2-550-48138-6

Marsan, J., Paré, G., \& Wybo, M.D. (2012). Has open source software been institutionalized in organizations or not? Information and Software Technology, 54, 1308-1316.

Ministère de la santé et des services sociaux (MSSS), (2013). Ensemble en réseau, pour vaincre le cancer. Plan d'action en cancérologie 2013-2015.

Paillé, P. (1994). Pour une méthodologie de la complexité en éducation : le cas d'une recherche-action-formation. Revue Canadienne de l'Éducation, 19(3), 215-230.

Pan, S.L., \& Leidner, D.E. 2003). Bridging communities of practice with information technology in pursuit of global knowledge sharing. Journal of Strategic Information Systems, 12, 71-88.

Sanchez, M.A., Vinson, C.A., La Porta, M., Viswanatah, K., ... Glasgow, R.E. (2012). Evolution of cancer control P.L.A.N.E.T. Application des résultats de la recherche. Cancer 1994, 23, 1205-1212.

South, A., Hanley, B.G., Gafos, M., Cromarty, B., ... Vale, C.L. (2016). Models and impact of patient and public involvement in studies carried out by the Medical Research Council Clinical Trial Unit at University College London: Findings from ten case studies. Trials, 17(376), 1-13. doi:10.1186/s1211-016-1488-9

Sylvain, H. (2008). Le devis constructiviste : une méthodologie de choix en sciences infirmières. L'infirmière clinicienne, 5(1), $11 \mathrm{p}$.

Woolf, S.H., Zimmerman, E., Haley, A., \& Krist, A. (2016). Authentic engagement of patient and communities can transform research, practice, and policy. Health Aff, 35(4), 590-594. 